\title{
Smart Building Creation in Large Scale HVAC Environments through Automated Fault Detection and Diagnosis
}

\author{
Maitreyee Deya ${ }^{\mathrm{a}}$, Soumya Prakash Rana ${ }^{\mathrm{a}}$, Sandra Dudley ${ }^{\mathrm{a}}$ \\ ${ }^{a}$ School of Engineering, \\ London South Bank University, London, United Kingdom
}

\begin{abstract}
Modernisation and retrofitting of older buildings has created a drive to install Building Energy Management Systems (BEMS) that can assist building managers in paving the way for smarter energy use and indirectly, using appropriate methods, occupant comfort understanding. BEMS may discover problems that can inform managers of building maintenance and energy wastage issues and in-directly, via repetitive data patterns appreciate user comfort requirements. The main focus of this paper is to describe a method to detect faulty Heating, Ventilation and Air-Conditioning (HVAC) Terminal Unit (TU) and diagnose them in an automatic and remote manner. For this purpose, a typical big-data framework has been constructed to process the very large volume of data. A novel feature extraction method encouraged by Proportional Integral Derivative (PID) controller has been proposed to describe events from multidimensional TU data streams. These features are further used to categorise different TU behaviours using unsupervised data-driven strategy and supervised learning is applied to diagnose faults. X-means clustering has been performed to group diverse TU behaviours which are experimented on daily, weekly, monthly and randomly selected dataset. Subsequently, Multi-Class Support Vector Machine (MC-SVM) has been employed based on categorical information to generate an automated fault detection and diagnosis system towards making the building smarter. The clustering and classification results further compared with wellknown and established algorithms and validated through statistical measurements.
\end{abstract}

Keywords: Heating, Ventilation and Air-Conditioning, Terminal Unit, Big Data, Feature Extraction, Machine Learning, Automatic Fault Detection and Diagnosis

\section{Introduction} more appropriate way depending on the time of day, year etc. Understanding large buildings play a vital role here, because they are now far more complex, energy consuming systems comprising several elements for example: Heating, Ventilation and Air-Conditioning (HVAC), lighting, power and control systems etc. These environments are also where people spend a vast amount of their day and finding ways to indirectly understand comfort drives energy profile changes in addition to in-house well-being initiatives [1].

Equipment failure and performance degradation of HVAC systems in commercial buildings often go unnoticed until such occurrences cause negative impact on occupant comfort, triggers an equipment-level alarm, deteriorates equipment life or results in excessive energy consumption and 
Overhead operational costs (OPEX). Therefore, buildings are installed with Building Energy Management System (BEMS), which are defined as an IT-based solution capable of sensing, control, and automation hardware to direct automated and/or manual improvements to system operations utilizing the multi-streams data. It is a rapidly rising market, with BEMS revenues for hardware, software and services anticipated to upswing from today's $\$ 2.7$ billion universal to $\$ 12.8$ billion by $2025[2]$.

This work focuses on the experimental application of machine learning in a real building's HVAC terminal unit (TU) data to detect and diagnose equipment failures, provide significant energy savings through pre-emptive maintenance, behaviour analysis and predictive building identification. Large volumes of rapidly generated TU data is always a challenging study, therefore, dedicated research is being carried out to analyse big data and develop a machine learning based approach to construct an Automatic Fault Detection and Diagnosis (AFDD) system. The algorithm employs a data driven approach and tie together the hidden information buried from big historical building data. The expected outcome of this research is remote TU fault identification and generating automatic notification to the building manager which leads to save the energy consumption.

\subsection{Literature Review}

HVAC systems have evolved enormously and significant development has been made in terms of data mining techniques for fault detection and diagnosis (FDD). HVAC research studies began in 1980's [3, 4], but from the beginning, practical limitations like scalability and complexity have made FDD extremely challenging. Based on this survey, the FDD techniques have been categorised into three major parts: model based, rule based and data-driven based approaches.

In model based approaches the data generated from plants and industries are simulations via mathematical models. In [5], physical laws were employed to derive the first principle model that signifies the dynamic nature of the system, which is not suitable for real time applications because such scenarios require fast controller responses. To further reduce the computational cost, a modified physical model was proposed by Shaw et.al [6]. Later this model incorporated real time HVAC applications $[7,8]$. There are a number of researchers who have proposed the gray-box model which is a non-complex model to reduce the computational cost of the system and few systems are proposed to handle the limitations of gray-box model $[9,10]$. A Black-box model was proposed for system identification based on mathematical modelling and has been found to be suitable for online FDD. Wu and Sun [11, 12] improved the performance of the black-box model for real time implementation based on parametric and multi-stage regression models, and were able to predict room temperature in office buildings.

Rule-based or Knowledge-based perspective for FDD in HVAC systems is applicable when there is insufficient data available to employ building mathematical models. This type of method is also applicable for large scale, complex HVAC systems, and requires a high level of expert knowledge on a system's fault features [13]. There are three different techniques: casual analysis, pattern classification, and expert systems that have been already developed for Air Handling Unit (AHU). Signed Directed Graph (SDG) is proposed for casual analysis by employing the fault symptoms without first principles [14]. The expert knowledge of a system can be implemented using some rules and the process can be divided into three types, (a) a system with partial knowledge by framing IF-THEN rules, (b) a system with deep-expert knowledge involved functional reasoning, and (c) systems with machine learning capabilities. Schein et. al., [15] developed a FDD system for operational control with partial or limited knowledge by setting up IF-THEN rules for an AHU. Manufacturers have effectively verified this concept for real time building applications [16, 17]. Schein and Bush [18] expanded the concept by proposing hierarchical rules for FDD. Yet, expert 
knowledge based techniques are limited when real data is unavailable. To resolve this restriction, machine learning methods like Hidden Markov Model (HMM) [19], Kernel Machines (KM) [20] are applied, where knowledge is automatically extracted from data. Pattern classification based algorithms are used to build non-linear correlations between data patterns and fault classes in the absence of clear model structures. Some popular pattern classification based methods are Bayes Classifier [21], Artificial Neural Networks (ANN) [20, 22], Support Vector Machine (SVM) [23], Fuzzy Logic [24].

Data-driven based techniques build relationships between data patterns and faulty classes of a system [25]. These approaches extract the key data components and transform the dimension of the entire data. These key components are then used instead of the whole dataset for FDD. This approach is appropriate for modern HVAC systems being used in huge commercial buildings. There are two types of categories for this approach: Signal based FDD and Multi-Variable Statistics (MVS) based FDD. Some existing signal based procedures are wavelet transformation and short-time Fourier analysis [26]. A combined method using wavelet transformation and principle component analysis (PCA) is proposed to diagnose faults for air handling unit (AHU) system [27]. It detects faults in large systems using a dimensionality reduction technique that maps the data to a lower dimensional space. In practice, this FDD methodology is appropriate for fault detection instead of fault diagnosis. To solve these limitations, integrated or hybrid approaches are considered for efficient FDD applications in large buildings [28].

All of these above stated approaches are limited to particular fault categories (e.g. fan failure, valve stuck) or fixed data. BEMS data analysis novella recent problem, indeed characterising Terminal Unit behaviour has not been given much attention in the research hitherto. Thus, this paper emphasises on TU behaviour analysis.

The paper is structured as follows: Section 2 outlines the motivation for this work and provides detail and context on Terminal Units and their associated problems. Section 3 focuses on the proposed methodology and imparts details about the feature extraction technique proposed along with the Clustering and Classification methodologies. Section 4 gives the discussion of experimental set-up and detailed data acquisition and analysis. The different TU behaviour patterns obtained as a result of the clustering and further classification result analysis with validation is presented in Section 5. Section 6 concludes the paper and provides the future research directions of this work.

\section{Structural Information of HVAC TU}

The data is collected for TU fan coil units (FCU) from a real building in central London, UK. The floor plan drawing of this building is observed closely to understand relationships between HVAC TUs, rooms, spaces, and other physical features at one level of a structure. The floor plan presented in Figure 1 shows the position of ground floor FCUs, which are marked with a red line border. There are 44 FCUs in the ground floor and the whole building comprises of 731 FCUs. These units are explicit subcomponents of HVAC systems set up in residential, commercial, industrial buildings and are responsible for the final delivery of comfort inside built environments.

A TU is a device consisting of a heating coil, cooling coil and a fan. It is normally ceilingmounted and monitored by local thermostats. It may either recirculate internal air, or introduce fresh air along with re-circulated air. Generally inside buildings, there is a central chiller and boiler plant that distributes cold water to all the cooling coils and hot water to all the heating coils. If the environment becomes too warm, the thermostat senses this and signals the chilled water valve, with cold water then passed through the coil, extracting the heat from the air being blown by the fan. If it gets too cold depending on the local set point, the heating coil start working in the same way. A typical schematic of a TU is shown in Figure 2. 


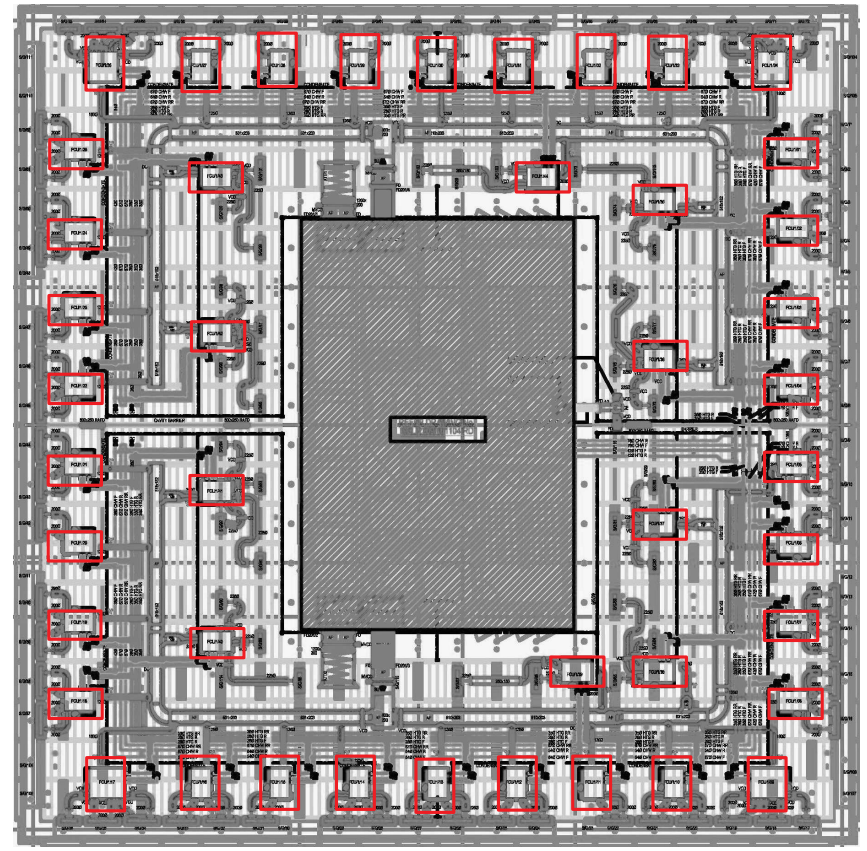

Figure 1: Floor plan architecture of a building in a central London

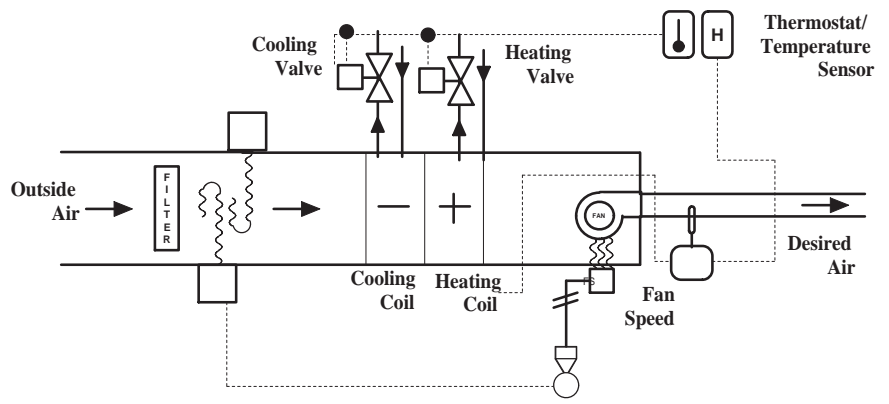

Figure 2: TU schematic diagram

The data thus gathered from the TUs inside building exhibit hidden information related to building behaviour. A single TU consist of various data streams are as follows:

- Control temperature $\left[{ }^{\circ} \mathrm{C}\right]$, frequently measured by each TU or in some cases a zone space temperature is used.

- Set point temperature $\left[{ }^{\circ} \mathrm{C}\right]$ is the desired or target control temperature of the unit is set by the operator or the administrator based on the current demand.

- Deadband is the control temperature band or range within the process and create two separate outputs of heating and cooling set point.

- Heating and cooling valve or damper actuator control and feedback signals.

- Enabled signal to indicate the hours of operation.

Poorly controlled or faulty TUs such as FCUs can be responsible for significant energy wastage and occupant discomfort. For example, a faulty fan coil unit can signal a false heating demand 
to the boiler, causing the ancillary equipment to activate and begin distributing hot water causing rooms to overheat. In the following subsection, different TU issues are explained which might cause faulty behaviour.

\subsection{Potential TU Issues}

There are a number of potential issues that can be identified from TU data analysis. Various behavioural metrics like saturation, on-ness and hunting can be studied for different TUs to identify the prevailing issues.

1. Saturation can be defined as the proportion of time over a day that the valve or damper is open at maximum. Thus the higher the value, the longer a heating or cooling valve (or damper) is open.

2. On-ness can be defined as the proportion of time that a terminal unit has any heating or cooling demand over a 24-hour period (i.e. any time that demand is greater than 0\%). Simply put, it is how long something is on.

3. Hunting is calculated using the set point, control temperature and is a measure of how much it fluctuates over a day.

Some of the potential issues that can result in faulty TU behaviours are listed as below:

1. Poor control- Typically, this can happen due to narrow dead bands being set and/or over aggressive proportional, integral and differential (PID) control. This can result in the TU frequently switching between heating and cooling.

2. Poor sensor location- The temperature sensor could be located at a wrong position. For an instance, in close proximity to heating and/or cooling elements (e.g. back of a drinks cabinet).

3. Varying set point- The set point may be varied too frequently typically due to occupant discomfort (e.g. user is changing set point for personal preference).

4. Out of hour operation- A unit may be found to operate out of hours because either it has simply been forgotten (manually operated and left) or the operational time schedule is incorrect.

5. Incorrect TU sizing for actual demand- It can happen that the load is underestimated and a bigger unit should have been installed. This is found more often in cooling than heating mode.

6. TU unable to receive adequate flow or upstream temperatures- The flow temperature from the boiler or chiller is not sufficiently high or low to condition the space, sometimes due to over-ambitious temperature compensation.

7. Stuck-open valve- Often indicated by a saturation value of 1 , it could mean the valve was fully open over a 24 -hour period.

8. Competition from nearby TUs- A TU is trying to heat the space but an adjacent TU near or in the same space, is trying to cool the same area. This is generally found where there is poor hierarchical control over a branch of TUs.

9. Localised effects- This can be caused either due to high solar gains or TU placed very close to energy-consumption equipment with high internal gains, like an old lighting fixture or photocopier.

10. Unachievable set point- Sometimes it can happen that a user adjusts the set-point temperature to maximum or minimum value that is simply unachievable for that environment.

Even though a TU is considered as "simple device" there can be a multitude of issues that can lead to faulty behaviour and require expert building engineering knowledge to identify and 
conclude each one of these issues. Manual TU data investigation can be extremely tedious and impossible with the ever-increasing amount of building data and the shortage of suitably qualified resident building engineers or managers. Hence automating and bringing intelligence to this process using data mining and machine learning approaches could be an ideal solution. This work therefore proposes a novel, data-driven feature extraction method and subsequent unsupervised clustering to identify different TU behavioural patterns without the need of an expert building engineer and further supervised classification employed towards making an automatic fault detection and diagnosis system for future data prediction.

\section{Proposed Methodology}

The current explosion of data in volume, recording and attributing, has initiated the expansion of many big data platforms along with parallel data analytics procedures. Huge data might produce worse performances in data analytics applications. Simultaneously, it has pushed for data dimensionality reduction procedures, but not always with better result [29]. The first milestone of the work was then to reduce the dimension of the data set. Thus, we emphasize a novel feature extraction method [30] for transforming the data into a lower dimensional space in a unique way.

Figure 3 shows the work flow involved here, comprising of two stages: unsupervised and supervised learning. Firstly, the vast data is held in a secure cloud and further applied feature extraction method, subsequently clustering is employed to group the similar patterns to identify separate faulty and non-faulty TUs. Subsequently, classification is applied through training and testing phases to understand fault trends and make useful predictions for fault diagnosis. This method leads to handle faults and reduce the energy wastage in a building.
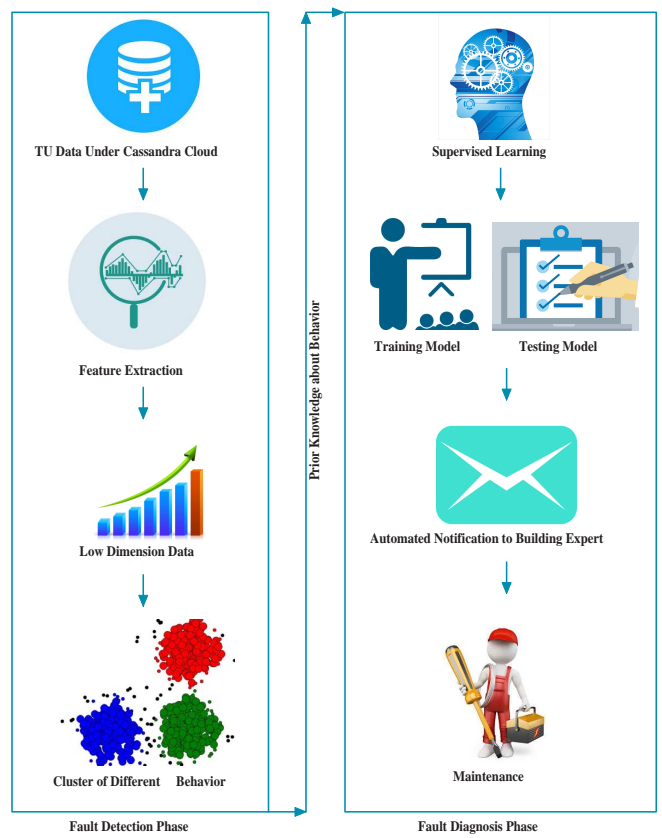

Figure 3: Computational steps involved in proposed methodology

\subsection{Feature Extraction using TU Data}

A novel feature extraction method is implemented based on the six different parameters of TU data such as, control temperature, set point, deadband, heating effort, cooling effort, and enable 
signal. The framework for feature extraction creates events by considering the area under the control temperature and the corresponding power curves which involves three different stages:

1. Event Discovery Stage

2. Event Area Calculation Stage

3. Event Aggregation Stage

\subsubsection{Event Discovery Stage}

The event discovery is inspired by the Proportional Integral Derivative (PID) controller response curve [31] as shown in Figure 4, a typical step response curve following a controller responds to a set point alteration. The curve rises within a period from $10 \%$ to $90 \%$ of the final steady state value known as the rise time. Percent Overshoot is the amount that the process variable overshoots the final value, expressed as a fraction of the final value. Settling time is the time required to settle within a certain percentage (commonly $5 \%$ ) of the final value. Steady-State Error is the ultimate difference between the process variable and set point. Dead time is a delay between when a process variable changes, and when that change can be observed. For instance, if a temperature sensor is placed far away from a cold water fluid inlet valve, it will not measure a change in temperature immediately if the valve is opened or closed. Dead time can also be caused by a system or output actuator that is slow to respond to the control command, for instance, a valve that is slow to open or close.

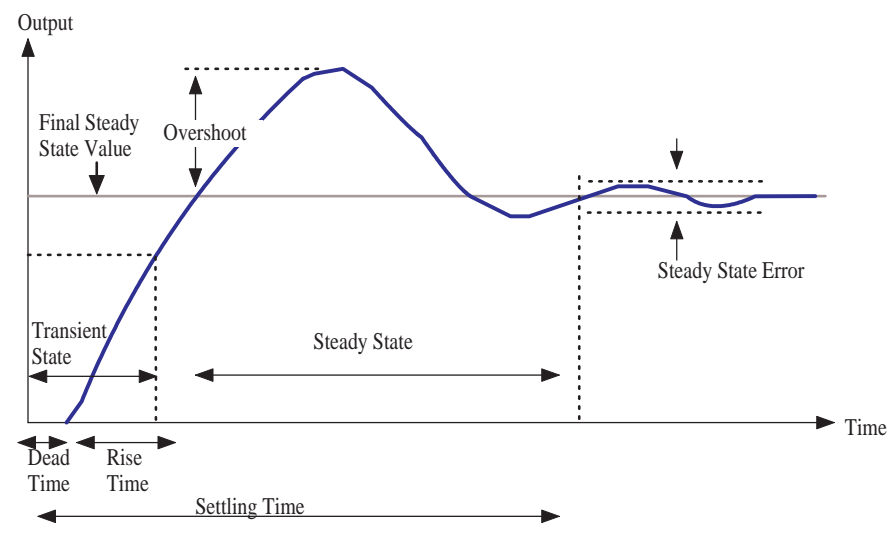

Figure 4: PID controller response curve

In this stage, the events are selected using control temperature and corresponding power effort data streams. Inside a building, when the heating and cooling units are started during normal operational hours, the temperature changes depending upon the environmental request. Therefore, two types of events, heating event and cooling event are selected depending on the corresponding power demand at that time instance. Based on the temperature variations with respect to the set point value, the data stream is divided into different phases and used to identify different events that happen in a single day. A single day TU data is being separated into four events as shown in Figure 5 .

1. Event Start (ES): ES is assumed when the BEMS starts and the time instance when temperature starts to change.

2. Response Delay (RD):The delay is anticipated when the temperature starts to respond only after a certain delay from the previous point when the BEMS starts. 
3. Goal Achieved (GA): When the temperature reaches to the desired set point, it is assumed as GA. This is basically the time during rise time.

4. Event End (EE): EE is occurring when the temperature exceeds the dead band.

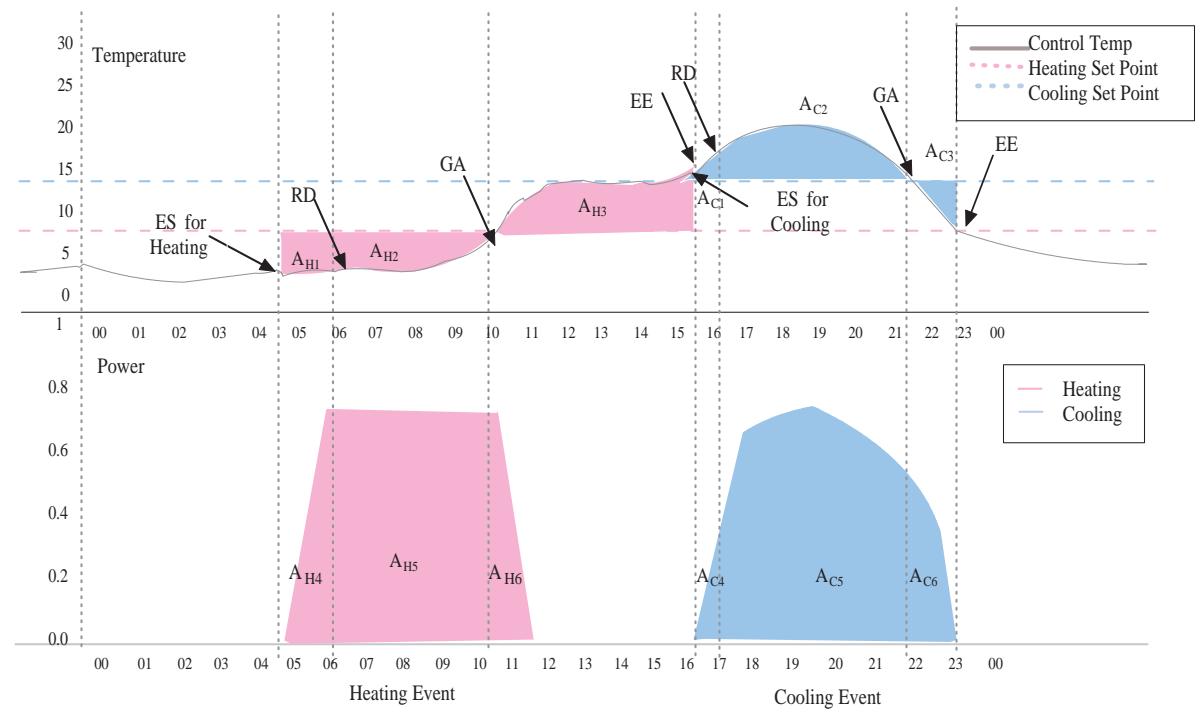

Figure 5: Event discovery process of one day TU

\subsubsection{Event Area Calculation Stage}

After discovering the appropriate heating and cooling events, the estimated area under the temperature and power curve for each event has been calculated. Subject to the event type area calculations are carried out respectively for both heating $(H)$ and cooling $(C)$ events. In effect, six areas (three from temperature and three from power curve) for each heating event and similarly, six areas for each cooling event are calculated. Altogether twelve different areas are derived from all the daily TU data.

Equation (1) shows the area $\left(A_{E}\right)$ beneath the curve $f(x)$ at every time interval $\Delta x$.

$$
A_{E}=\sum_{i=0}^{n} f\left(x_{i}\right) \Delta x
$$

In heating event, the area calculations for temperature are indicated by $A_{H_{1}}$ to $A_{H_{3}}$ and for power indicated by $A_{H_{4}}$ to $A_{H_{6}}$. Likewise for a cooling event, the area calculations for temperature are indicated by $A_{C_{1}}$ to $A_{C_{3}}$ and for power are indicated by $A_{C_{4}}$ to $A_{C_{6}}$. After these areas have been computed, they are normalized to obtain the final feature values as $F_{H_{1}}$ to $F_{H_{6}}$ and $F_{C_{1}}$ to $F_{C_{6}}$ as denoted by Equations (2) to (5).

Equations (2) and (3) show the area calculations for a heating event.

$$
\begin{array}{r}
F_{H_{1}}=\frac{A_{H_{1}}}{T_{H_{1}}}, \quad F_{H_{2}}=\frac{A_{H_{2}}}{T_{H_{1}}}, \quad F_{H_{3}}=\frac{A_{H_{3}}}{T_{H_{2}}} \\
\text { where, } T_{H_{1}}=\max \left(A_{H_{1}}+A_{H_{2}}\right) \\
\text { and, } T_{H_{2}}=\max \left(A_{H_{3}}\right)
\end{array}
$$




$$
\begin{array}{r}
F_{H_{4}}=\frac{A_{H_{4}}}{P_{H_{1}}}, \quad F_{H_{5}}=\frac{A_{H_{5}}}{P_{H_{1}}}, \quad F_{H_{6}}=\frac{A_{H_{6}}}{P_{H_{2}}} \\
\text { where, } P_{H_{1}}=\max \left(A_{H_{4}}+A_{H_{5}}\right) \\
\text { and, } P_{H_{2}}=\max \left(A_{H_{6}}\right)
\end{array}
$$

Equations (4) and (5) show the area calculations for a cooling event.

$$
\begin{array}{r}
F_{C_{1}}=\frac{A_{C_{1}}}{T_{C_{1}}}, \quad F_{C_{2}}=\frac{A_{C_{2}}}{T_{C_{1}}}, \quad F_{C_{3}}=\frac{A_{C_{3}}}{T_{C_{2}}} \\
\text { where, } T_{C_{1}}=\max \left(A_{C_{1}}+A_{C_{2}}\right) \\
\text { and, } T_{C_{2}}=\max \left(A_{C_{3}}\right) \\
F_{C_{4}}=\frac{A_{C_{4}}}{P_{C_{1}}}, \quad F_{C_{5}}=\frac{A_{C_{5}}}{P_{C_{1}}}, \quad F_{C_{6}}=\frac{A_{C_{6}}}{P_{C_{2}}} \\
\text { where, } P_{C_{1}}=\max \left(A_{C_{4}}+A_{C_{5}}\right) \\
\text { and, } P_{C_{2}}=\max \left(A_{C_{6}}\right)
\end{array}
$$

\subsubsection{Event Aggregation Stage}

Multiple heating and cooling events can occur during a single day therefore, all the events of a given type need to be aggregated to represent the averaged values. So, the next step in the feature extraction process is event aggregation. The final aggregated features can be represented using the Equations (6) and (7), where, $k$ denotes the number of events and $n$ denotes total number of occurrences for each event in both the type. Thus, a single day TU data can be represented using twelve features for both the heating and cooling events.

$$
\begin{aligned}
& F_{H_{k}}=\frac{1}{n} \sum_{i=1}^{n}\left(F_{H_{k_{i}}}\right) \\
& F_{C_{k}}=\frac{1}{n} \sum_{i=1}^{n}\left(F_{C_{k_{i}}}\right)
\end{aligned}
$$

\subsection{Unsupervised Learning}

Due to the lack of prior knowledge about the data involved, an unsupervised learning methodology is employed to discover a set of TU behaviours. Here, an extended K-Means (X-means) [32] clustering is employed on the extracted TU features (obtained from the Equations (6) and (7)). This clustering is used to avoid the limitation of conventional K-means clustering, which tried to automatically determine the number of clusters based on Bayesian Information Criterion (BIC) scores. Initially, the whole dataset is considered as single cluster and BIC score is calculated by Equation 9. Now, the conventional $K$-means is performed by varying cluster seed value. The following steps of $X$-means are as follows:

Improve-params (k-means)- $K$-means is evaluated by the seed value of cluster number. The objective function used for this work is the Equation 8.

$$
J=\sum_{j=1}^{k} \sum_{i=1}^{n}\left\|X_{i}^{(j)}-\mu_{j}\right\|^{2}
$$


Where, $\left\|X_{i}^{(j)}-\mu_{j}\right\|^{2}$ is the Euclidean distance measure between a data point $X_{i}^{(j)}$ and the cluster centre $\mu_{j}$. It is an indicator of the distance of the $n$ number of data points from their respective cluster centres. The main idea is to define $K$ centroids, one for each cluster. Each data point is assigned to the group that has the closest centroid. After all the data points are assigned, the positions of the $K$ centroids are recalculated. The above steps are reiterated till the centroids no longer move.

Improve-structure (BIC)- In this stage, the BIC score is calculated based the clustering outcomes. Maximum likelihood of the current clusters is used to determine the BIC. Centroids are broken further based on the BIC score in order to better fitting of the data. The BIC is computed here using Equation 9.

$$
\begin{array}{r}
B I C_{\text {score }}=-2 \log (L)+K \log (n) \\
\text { where, } L=P(x \mid \theta, M)
\end{array}
$$

Where, $L$ is the maximum value of the likelihood function of the model $M$. The other parameters, $x, \theta, n$, and $K$ denote the observed data, the parameter of the model, total number of data points and the number of free parameter to be estimated respectively.

$\mathbf{K}>\mathbf{K m a x}$ - Once centroid $(k)$ is determined and $K$-means is performed, $K_{\max }$ is then selected, and all centroids are tested. As the lower BIC score is always preferred for better fitness of the data, then BIC score is compared between $K$ and $K_{\max }$. If the current model has a better score, then the split is considered the best strategy for clustering.

\subsubsection{Clustering Internal Evaluation}

Internal validation criteria are used to evaluate the effectiveness of an unsupervised learning technique where the external known class labels are not present. This evaluation measures the degree of intra-cluster cohesion and inter-cluster separation. Here, Davies-Bouldin (DB) and Silhouette (SI) criterion is perform for the evaluation of the clustering technique.

- Davies-Bouldin Index- The Davies-Bouldin index is defined in Equation 10, where, $\Delta_{i, j}$ is the clusters distance ratio for the ith and jth within to between cluster. $\partial_{i}$ and $\partial_{j}$ is the average distance between each point in the cluster from the centroid of that $i t h$ cluster and the average distance between each point in the $j t h$ cluster and the centroid of the $j t h$ cluster [33].

$$
D B=\frac{1}{k} \sum_{i=1}^{k} \max _{j \neq i} \frac{\delta_{i}+\delta_{j}}{\Delta_{i, j}}
$$

- Silhouette Index- The silhouette indexing [34] is a measure of the similarity of each point with other points in its own cluster, when compared to points in other clusters. The silhouette value is defined in Equation 11. Here $a_{i}$ is the average distance from the $i t h$ point to the other points in the same cluster, and $b_{i}$ is the minimum average distance from the $i t h$ point to points in a different cluster.

$$
S I=\frac{1}{n k} \sum_{i \in k} \frac{b_{i}-a_{i}}{\max _{\left(a_{i}, b_{i}\right)}}
$$

The silhouette value ranges from -1 to +1 . A high value indicates that $i$ is well-matched to its own cluster. The clustering solution is considered appropriate if most points have a high silhouette value. Whereas, a low DB value consider the clustering solution is appropriate. 


\subsection{Supervised Learning}

Supervised learning is exercised on the discovered knowledge from clustering. It has training and testing phases to adopt and predict different faults. Although several methods are adopted for classification, support vector machine (SVM) is a well-known binary classifier but it is modelled as a multiclass classifier in the work presented here. Thus, Multi-Class Support Vector Machine (MC-SVM) $[35,36]$ is employed on the extracted TU feature by optimizing the distance between support vectors (TUs from different groups) given by Equations (12) and (13).

In Equation (12) a set of training pattern is denoted by $\left(x_{1}, y_{1}\right), \ldots,\left(x_{l}, y_{l}\right)$ of cardinality $l$, where $x_{i} \in R^{d}$ and $y_{i} \in 1, \ldots, k, w \in R^{d}$ is the weight vector, $C \in R_{+}$is the regularization constant, and $\varphi$ is mapping function which projects training pattern into a suitable feature space $H$ that allows for nonlinear decision surfaces. The constraints $\xi_{i} \geq 0, i=1, \ldots, l$, are implicitly indicated in the margin constraints of (12) when $t$ equals $y_{i}$.

$$
\begin{gathered}
\min _{w_{m} \in H, \xi \in R^{l}} \\
\text { subject to } \quad w_{y_{i}}^{T} \varphi\left(x_{i}\right)-w_{t}^{T} \varphi\left(x_{i}\right) \geq 1-\delta_{y_{i}, t}-\xi_{i} \\
i=1, \ldots, l, t \in 1, \ldots, k
\end{gathered}
$$

Whereas in Equation (13), $\delta_{i, j}$, is the delta (defined as 1 for $i=j$ and as 0 otherwise). The final decision function is defined as,

$$
\operatorname{argmax}_{m} f_{m}(x)=\operatorname{argmax}_{m} w_{m}^{T} \varphi(x)
$$

In addition Equation (12) focuses on classification rule (13) without any bias terms. A non-zero bias term can be simply exhibited by adding an additional feature to each $x$. Therefore, different categories of data are classified by solving this decision function and the results are analysed in the following section. A pseudo code for the proposed work is presented in the Algorithm 1.

\subsubsection{Classification Performance Evaluation}

After performing classification the obtained results are evaluated through well-known statistical performance metrics, precision and recall for calculating the accuracy of the system. Precision calculate the percentage of truly predictive TUs among all the positively detected TUs in the dataset and recall measures the relevant outcomes which are detected among the total dataset shows in Equations (14) to (15). The performance measurement is based on the number of true positive (TP), false positive (FP), and false negative (FN). TP is the total number of correctly labelled TUs, whereas FP is the total of incorrectly assigned TUs, and FN is the total of not labelled as should be belonging to particular classes.

$$
\text { Precision }=\frac{\text { TruePositive }}{\text { TruePositive }+ \text { FalsePositive }}
$$

$$
\text { Recall }=\frac{\text { TruePositive }}{\text { TruePositive }+ \text { FalseNegative }}
$$




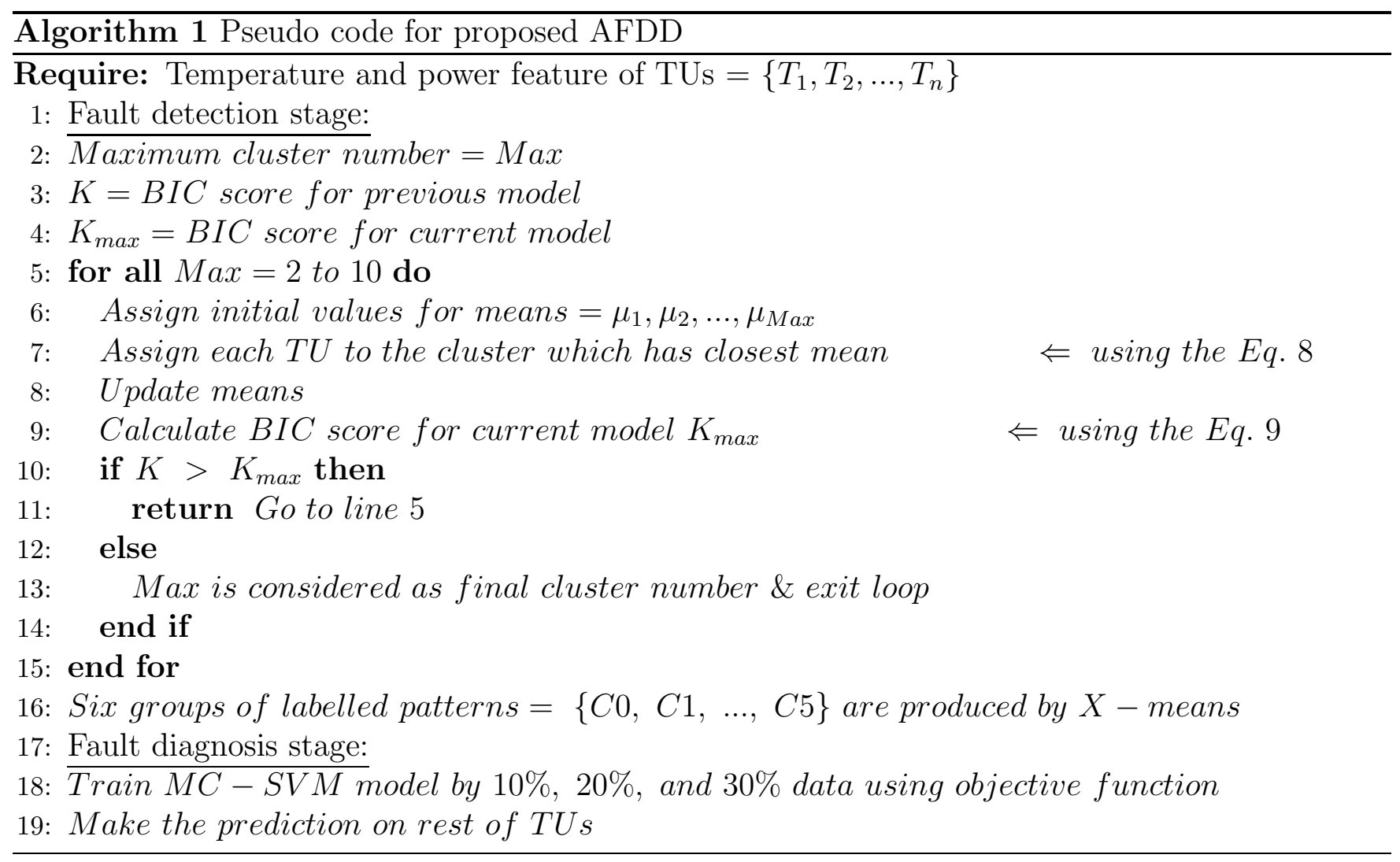

\section{Experimental Set-Up}

The present case study is a seventeen floor building in central London, U.K. with seven hundred and thirty one terminal units distributed across the different floors. Figure 6 shows the developed system architecture and various stages of our proposed AFDD, which handles data storage, retrieval, analysis, visualization, and support for decision making. There are six different parameters (set point, dead band, control temperature, enabled, heating effort, cooling effort) selected for this experiment. Based on these parameters the demand and the control strategies are varied and deal with the real time TU issues as mentioned in section 2.1. These raw data comprise information e.g. time stamp, voltage, temperature, etc. It has problems, as would be expected in real environments, of different sampling rates for different attributes, variable arrival time, missing values for intermediate time stamps, etc. Therefore, the TUs with the missing data are filtered and linearly interpolated to resample data at regular ten minute time intervals.

\subsection{TU Data Retrieval Process}

The BEMS data is extracted via a single embedded PC connected to the BEMS network as a network node (thus the device needs to be given an address on the network - the detail of this depends on the type of BEMS). Currently a PC Engine 2D13 ALIX embedded PC is used. The embedded PC is also connected to the Internet using either the buildings existing Internet connection or through a mobile network router.

\subsubsection{Data Collection}

The embedded PC contains embedded software that is used to:

1. Obtain a map of the BEMS network. This includes all the: 


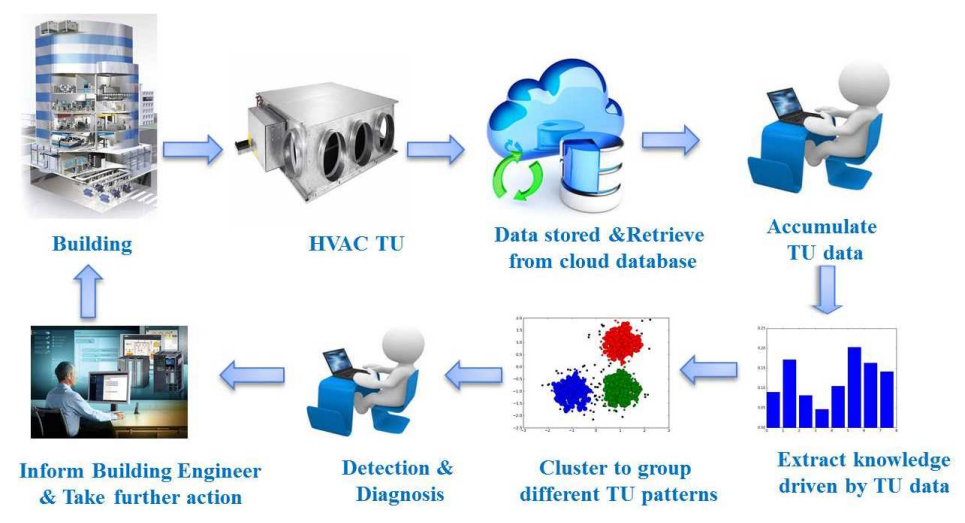

Figure 6: Framework of the experimental TU data retrieval process

- Local Area Networks (LANs) on a BEMS network (a BEMS network is more accurately called internetworks and consists of multiple LANs).

- Devices on a LAN (a single device may relate to one or many pieces of building services equipment).

- Data points on a device. These can be binary or analogue control signals, feedback signals or settings.

For each of the LANs, Devices and Data point the text label and numerical ID is obtained.

2. Polling the values of the data points (typically at intervals of 30 minutes data point type and, by inference, how frequently values are likely to change. For example: the control and feedback signals are polled more frequently while the settings are polled less frequently).

3. Store/buffer the data if the internet connection is lost.

4. Securely send the data to the cloud servers.

\subsubsection{Data Characteristics}

This BEMS data follows the characteristics of " $4 V$ " [37], i.e., volume, variety, velocity, and value which reflects big data characteristics.

Volume: The data is continuously generating and increasing the volume. In this experiment, approximately 48 million data points are considered.

Variety: The data type is not only traditional structured, but also mixed of structured, semistructured, and unstructured. There are six types of data are included such as, time stamp, voltage, temperature, binary signal on and off. This complete set of different attributes make a composite structure which needs a complex processing logic to handle the aggregation.

Velocity: The speed of the data flow is very fast. These massive and continuous data is stored in Cassandra cloud from the source device. Approximately one million data points are gathered every day for a single building, which helps us to make valuable decisions that provide strategic advantages after handling the data velocity.

Value: These data are as valuable as the building outcomes. It enables us to be aware of the behaviour of TUs, which increase the potential to improve fault analysis, decision making capabilities and measure the faulty equipment for diagnosis and energy saving purpose. The proposed work is implemented feature extraction, clustering and classification for decisionmaking process and to determine the ultimate value of the collected data. 


\subsection{Typical Lab Set up for Parallel Processing}

The implementation and performance of the proposed method relies on the processing architecture in place and is shown in Figure 7. The architecture has been set-up for this work comprises Apache Spark [38] an open-source cluster computing framework that enables the scalability and fault tolerance of Map Reduce using resilient distributed datasets (RDDs). A RDD is a group of objects partitioned through a set of machines that can be reconstructed if partition is lost. Spark in memory runs up to 100 times faster for certain applications by allowing user programs to load data into a clustered memory and querying repeatedly. It is highly suitable for machine learning algorithms [39, 40]. Spark requires a cluster manager and a distributed storage system. Here Spark version 2.0 is used with $5 \mathrm{CPU}$ core of $8 \mathrm{~GB}$ memory each is set-up by one master and four workers and the connection of the distributed storage with Apache Cassandra interface. The experiments have been performed using 64-bit Java version 8 .

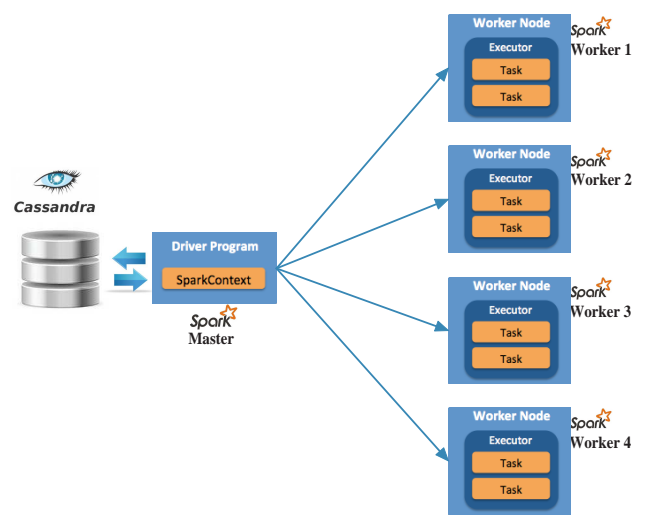

Figure 7: Set-up of parallel processing architecture

\section{Result Analysis}

After cleaning and aggregating the data as discussed in the previous section, experiments were conducted on four types of data volumes such as, daily, weekly, monthly and randomly selected data from the months of July to October (inclusive) 2015. The analysis has been performed on the building under test's historical data to reveal hidden patterns through the employment of machine learning. Day analysis was performed on 17th July 2015 TUs, week analysis performed from 17th July to 23rd July for 5 working days (excluding weekend) and month analysis done for 22 days since weekends have been excluded from the analysis. The random analysis was conducted on the randomly selected TUs from July to October 2015. Data details are given in Table 1.

Table 1: Experimental data details

\begin{tabular}{|l|l|l|l|}
\hline No of Days & $\begin{array}{l}\text { Total number } \\
\text { of TUs }\end{array}$ & $\begin{array}{l}\text { Operating } \\
\text { TUs }\end{array}$ & Description \\
\hline 1 Day & 731 & 723 & 17th July, 2015 \\
\hline 1 Week, (5 days) & 3655 & 3615 & $\begin{array}{l}\text { 17th to 23rd July 2015 } \\
\text { (except 18th and 19th) }\end{array}$ \\
\hline 1 Month, (21 Days) & 15351 & 15178 & $\begin{array}{l}\text { 17th July to 14th August 2015 } \\
\text { (except weekends) }\end{array}$ \\
\hline 3 Months, (71 Days) & 39088 & 38591 & $\begin{array}{l}\text { 17th July to 23rd October 2015 } \\
\text { (except weekends) }\end{array}$ \\
\hline
\end{tabular}


A new feature extraction algorithm (presented in Equation (2) - (5)) is proposed to reduce, measure, and build derived values from these initial datasets intended to be informative and nonredundant, which is facilitates subsequent learning and generalization steps. The raw data is transformed into a 12-dimensional $\left(\left(F_{C_{1}}\right.\right.$ to $\left.F_{C_{6}}\right)$ and $\left(F_{H_{1}}\right.$ to $\left.\left.F_{H_{6}}\right)\right)$ data for each TU. The visualization of these multivariate features for a single TU are illustrated by radar in Figure 8. The radar graph is a circular display with twelve different quantitative axes. Each axis represents a single feature which signifies the fluctuation of temperature and power. The centre of the radar is zero valued and the edge point is with maximum value. These feature values are plotted along each axis that forms a unique shape and describes TU performance. The proposed feature extraction algorithm (described in section 3.1) generates a feature vector of 12 numeric values which contains the cooling and heating behaviour of a TU. Thus, the radar is being divided into two parts, first six axes $\left(\left(F_{C_{1}}\right.\right.$ to $\left.\left.F_{C_{6}}\right)\right)$ are represent cooling events and next six axes $\left(\left(F_{H_{1}}\right.\right.$ to $\left.\left.F_{H_{6}}\right)\right)$ are represent heating events.

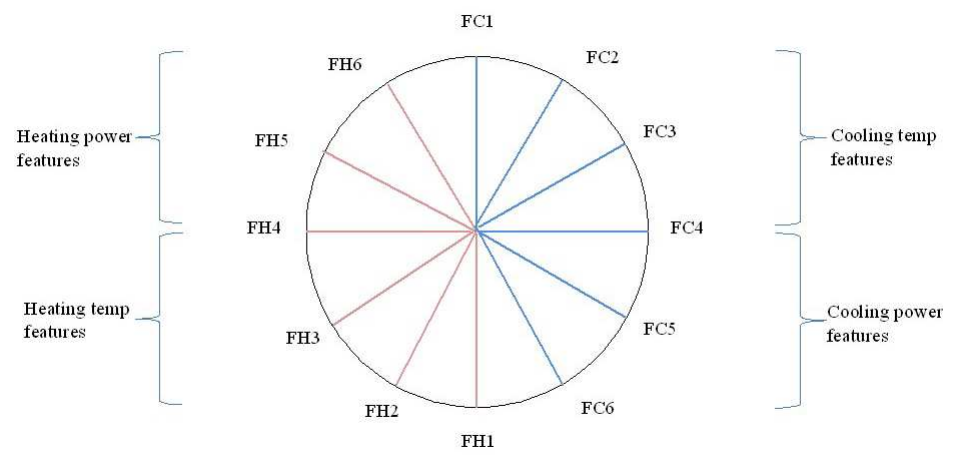

Figure 8: Description of radar graph

Here, $F_{C_{1}}$ and $F_{H_{1}}$ describe the temperature state through the area from the event start (ES) to response delay (RD), $F_{C_{2}}$ and $F_{H_{2}}$ describe the time of response through the area from response delay (RD) to goal achieve (GA), $F_{C_{3}}$ and $F_{H_{3}}$ describe the event end after reaching the set-point through the area from goal achieve (GA) to event end (EE). Similarly, $F_{C_{4}}$ to $F_{C_{6}}$ and $F_{H_{4}}$ to $F_{H_{6}}$ represent the power demand for corresponding temperature change. So, it is to be further noted that greater areas under GA to EE area represent a "well behaved" TU, because this implies that the TU achieves the set point (goal) and spends more time within the dead band (range between heating and cooling set point), whereas the area under ES to RD and RD to GA is larger, it denotes that the temperature is not within the dead band or takes a long time to reach the set point. The more area for power effort indicates the more power consumption which also signifies a "badly behaved" TU.

\subsection{Clustering Analysis}

Now, the TU data with different attributes are present, but the characteristics or possible patterns are unknown except for functioning and malfunctioning TUs. Therefore, the X-means clustering is performed to find out the possible partitions of the data by varying cluster number from 2 to 10 with 6 being found as the optimal number of cluster to describe the distinct TU behavioural patterns.

Clustering is performed on approximately forty thousand TUs and the visualization of the compact groups is shown in the radar graphs below. Figure 9 shows six radars of the daily clustering analysis and each radar represents a single TU which is the nearest to that cluster centre to make 
a generalized conclusion on that group. It is discovered from cluster analysis of TU characteristics pattern over daily data (shown in Figure 9), that the heating trend is captured in cluster-3 and cooling trends are captured in rest of the clusters. TUs belonging to cluster-0 achieve their set point and the feature values are in the third axis $\left(F_{C_{3}}\right)$ of the radar. It represents the area under GA to EE events for the temperature curve, therefore these TUs exert little power effort and stay within their dead band. Cluster-1 represents TUs whose features are in the third axis $\left(F_{C_{3}}\right)$ which indicates GA to EE state i.e., those TUs require average power (e.g., values are in $F_{C_{6}}$ axis) to reach the set point. So, TUs belong to cluster-1 use extra power than the TUs are in the cluster-0. The TUs of cluster-2 behave similar to cluster-1 but these TUs use higher power levels to reach the set point within the buildings operational hours. TUs of cluster-3 capture the area under RD to GA $\left(F_{C_{5}}\right)$ is large for both the temperature and power curves, which means more heating power is required, and the set point is not achieved during working hours. The feature values of cluster- 4 fit in first axis $\left(F_{C_{1}}\right)$ which implies time requirement is very large. Thus, area under the ES to RD state for both the temperature and power curves indicates that the TUs take longer times and are unable to achieve the required set points. For cluster-5, the area under the RD to GA for both the temperature and power curves shows the need of high power.
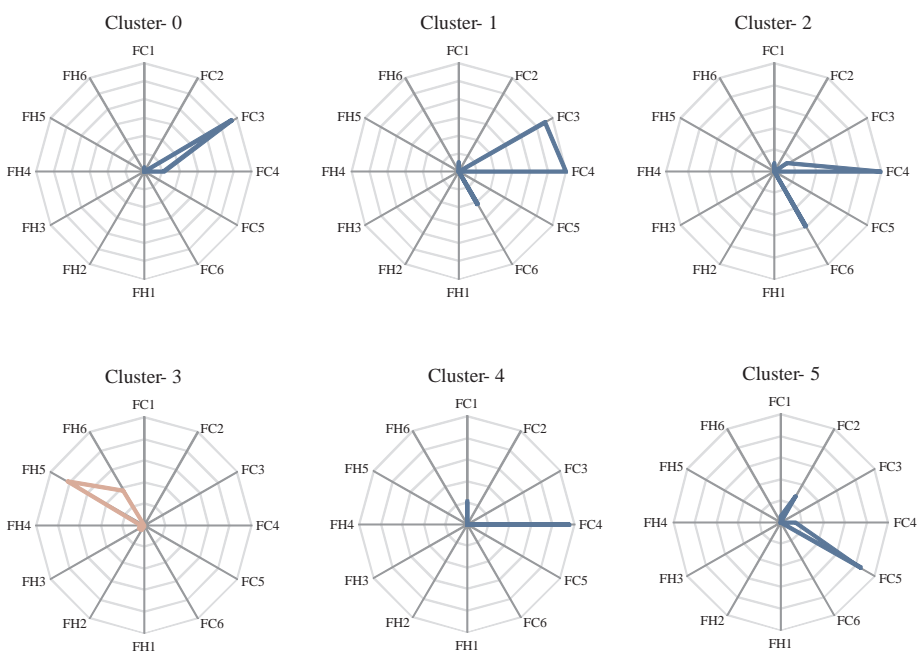

Figure 9: Cluster analysis of Daily TUs

Two examples of faulty and non-faulty deemed TUs, obtained from the cluster analysis are shown in Figure 10. Figure 10(a) shows cooling non-faulty TU behaviour which belongs to cluster-0 of Figure 9. This indicates the behaviour where the temperature is consistently held within the deadband and low power is consumed during working hours. Figure 10(b) shows a cooling faulty TU behaviour which belongs to cluster-4 of Figure 9. Though the TU is exerting maximum effort in terms of power consumption, it is still unable to reach the desired set point. In this case it may be that the TU is indeed faulty or that the TU is not actually faulty but that the requests made of it are unreasonable i.e. poor sensor location (behind a drinks dispense cabinet for example), the room is sun drenched or has large unopened windows. Pivotally, the data shows there is a problem. Moreover, this work is not limited to any fixed type of faults; it can detect any kind of abnormal behaviour as faulty, where the temperature and power are both or either in an untenable position as is the case here.

Therefore, the X-means clustering partitioned the daily TU data into 6 groups based on their property. It revealed the 6 different hidden patterns of the TUs. These distinct behaviours are 


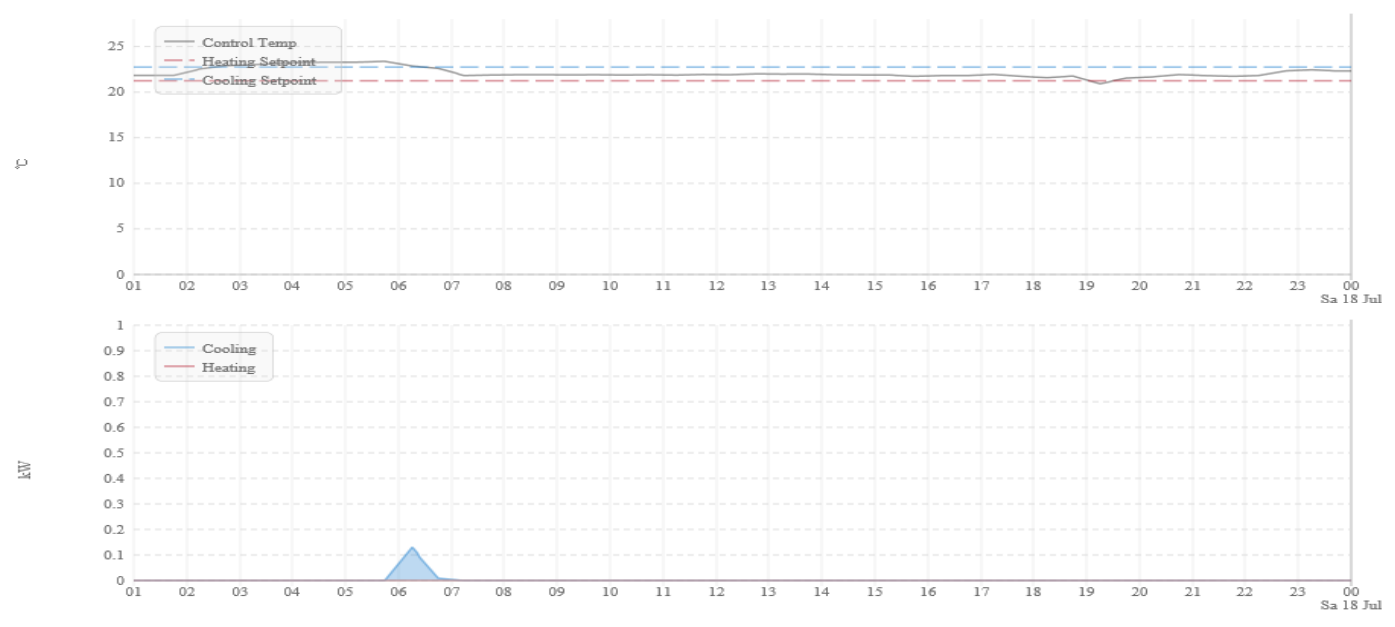

(a) Temperature within dead band with low cooling power

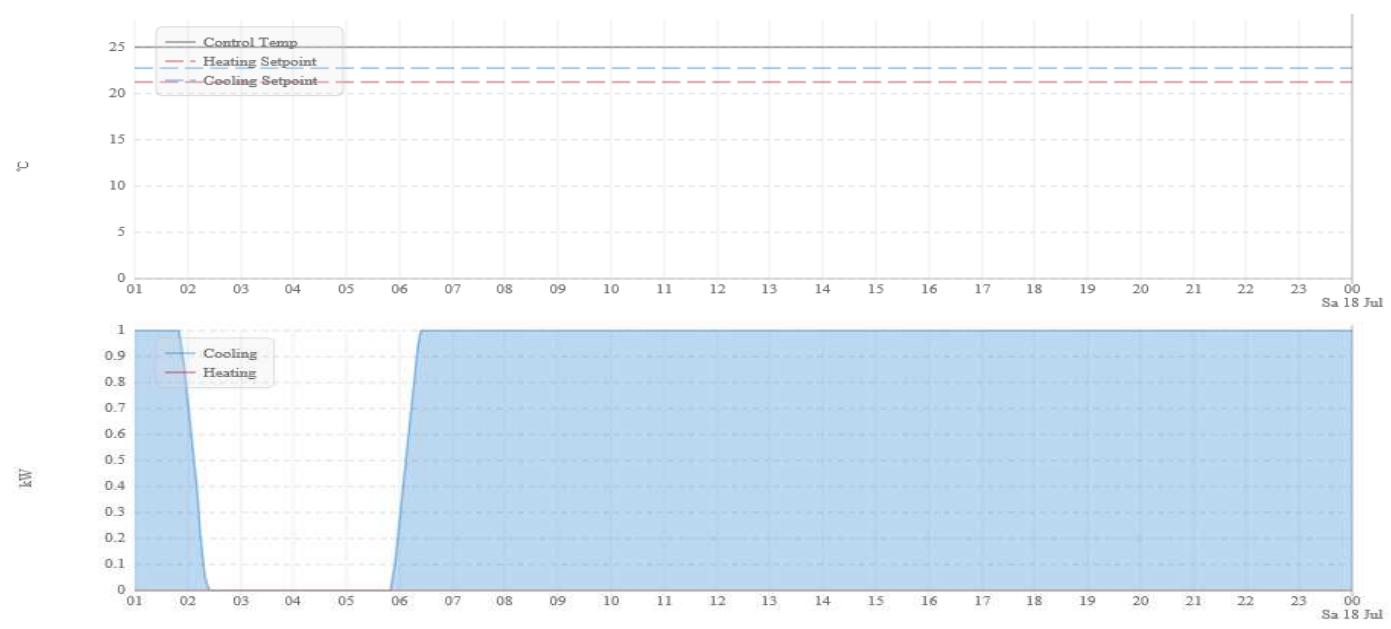

(b) Cooling saturation and on-ness

Figure 10: Example of (a) a non-faulty TU and (b) a faulty TU

Table 2: Cluster pattern description of daily TUs

\begin{tabular}{|l|l|}
\hline $\begin{array}{l}\text { Cluster } \\
\text { Number }\end{array}$ & Description of Clustering Pattern \\
\hline C-0 & $\begin{array}{l}\text { TUs that show both heating and cooling characteristics. The desired } \\
\text { control temperature is achieved with low power effort, } \\
\text { and is done in normal building operating hours. }\end{array}$ \\
\hline C-1 & $\begin{array}{l}\text { These are cooling TUs where the control temperature is achieved } \\
\text { within the desired band with medium or average power effort. }\end{array}$ \\
\hline C-2 & $\begin{array}{l}\text { Cooling TUs where control temperature is achieved but with a } \\
\text { high-power effort. }\end{array}$ \\
\hline C-3 & $\begin{array}{l}\text { TUs showing control temperature hunting patterns along with medium } \\
\text { to high power effort and continuation of this pattern outside operational hours. }\end{array}$ \\
\hline C-4 & $\begin{array}{l}\text { TUs where the control temperature does not achieve the desired set point } \\
\text { (out by up to 5 degree C) with high power effort. }\end{array}$ \\
\hline C-5 & $\begin{array}{l}\text { TUs where the control temperature does not achieve the desired set point } \\
\text { (out by up to 10 degree C) with high power effort. }\end{array}$ \\
\hline
\end{tabular}


Furthermore, this experiment is conducted on the same number of TUs for a week, month, and for random data to check the feasibility, and robustness of the proposed clustering method. Figure 11(a) shows the cluster analysis of TU behaviours during week days, 11(b) for a month, and 11(c) for randomly selected TUs. The repetition of the TU behaviour is found during the clustering experiment on weekly, monthly, and randomly selected data. TUs of cluster-0 of Figure 9, cluster-0 of Figure 11(a), and cluster-3 of Figure 11(b) are found to have similar heating and cooling trends, where goal or desired temperature is achieved with very low power demand. Likewise, the TUs of cluster-1 of Figure 9, cluster-2 of Figure 11(a), cluster-0 of Figure 11(b), and cluster-0 of Figure 11(c) follow similar trends where the set-point is achieved with average power effort. In case of TUs from cluster-2 of Figure 9, cluster-5 of Figure 11(a), and cluster-2 of Figure 11(c) higher power effort are needed to reach the set-point. The hunting behaviour of TUs is found with high power consumption during operational and out of operational hours in cluster-3 of Figure 9, cluster-1 of Figure 11(a), cluster-1 of Figure 11(b), and cluster-3 of Figure 11(c). Likewise the daily TUs of cluster-4 of Figure 9 is similar to cluster-3 and cluster-5 of Figure 11(a) and Figure 11(b), where set-point is not achieved but power is still consumed during out of operational hours. Similar TU behaviours e.g. cluster- 5 of Figure 9 is found respectively in cluster-4, cluster- 4 , and cluster- 5 of Figure 11(a), 11(b), and 11(c). It is noted that the patterns found in cluster-2 of Figure 11(b) and cluster-4 of Figure 11(c) are very similar to each other, where set-point is achieved and both captured heating trends. Another two similar heating patterns of TUs are found in cluster-1 and cluster-3 of Figure 11(c), both achieved set-point using average to high power effort.

\subsubsection{Clustering Validation}

This initial pattern discovery process is completely unsupervised where no prior label information is available. Internal cluster evaluation criteria (Davies-Bouldin and Silhouette) are used to validate the clustering results and assess compactness of those groups. Also, the proposed X-means results are compared with two different clustering algorithms: Hierarchical clustering and Gaussian mixture model [41] to prove the advantage for using X-means. The quality of the clustering and reason to use $\mathrm{X}$-means is summarized in Figure 12. The comparison and validation results across daily, weekly, monthly and randomly selected TU data are included here.

Figure 12(a) demonstrates the DB indexing results of X-means for cluster number 2 to 10 and compared with hierarchical and Gaussian mixture algorithm. As the lowest DB value is preferred, it is seen from the graph that lowest DB value is found when the cluster number is six for the daily, weekly, monthly, and randomly selected TUs for x-means. Similarly, DB values are little higher in case of hierarchical clustering. But heavy fluctuation of DB values of Gaussian mixture model shows the incompactness of the clustering outcomes. Therefore, the lower value of DB in $\mathrm{X}$-means for cluster number 6 shows a good compactness of the clusters than hierarchical clustering. Figure 12(b) shows the SI indexing results for x-means, hierarchical and Gaussian mixture algorithm. The clusters with high SI index are well matched to its own cluster. It is clearly seen that the SI index is quite high for x-means on daily, weekly, monthly, and randomly selected TU data, when the cluster number is six, whereas hierarchical clustering achieved high SI indexing for cluster number six in all cases except randomly selected TUs. Again, in the Gaussian mixture algorithm, SI values are not concentrated for a single cluster number, therefore it is hard to discover a cluster number and their compactness. These internal clustering validation results shows that the selected optimal number of clusters is appropriate and achieved suitable indexing outcomes consistently for x-means than other clustering algorithm. These clustering behaviours results for the developed AFDD were all further verified across all tested HVAC TUs with the help of expert building engineers from Demand Logic. 

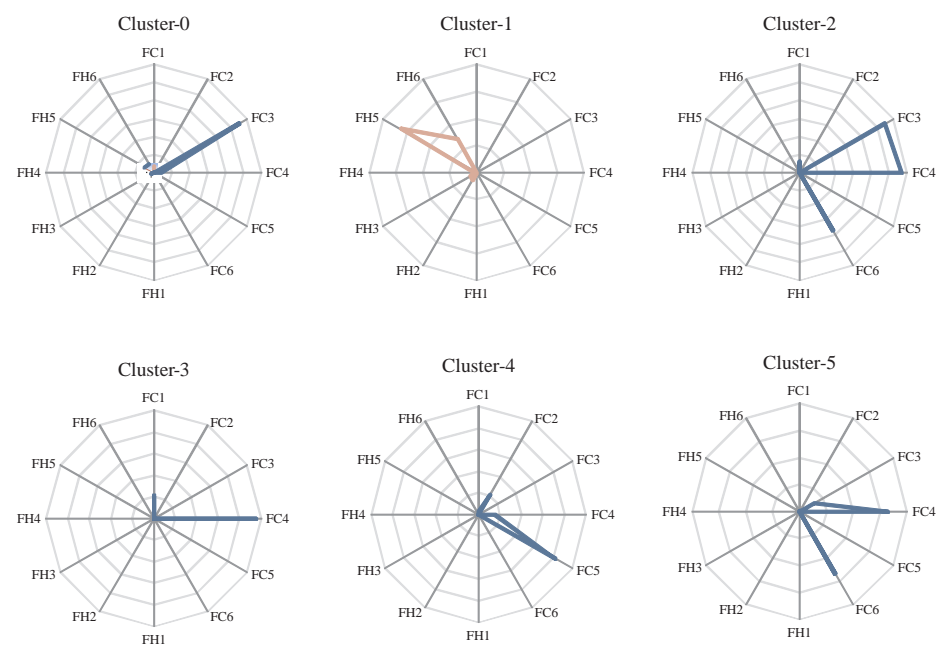

(a) Weekly Cluster Radar
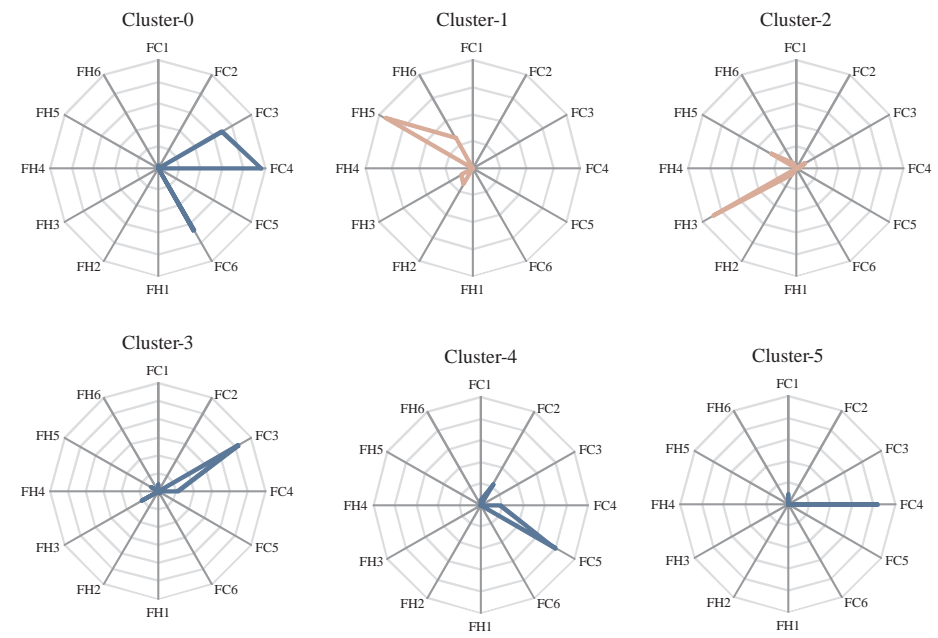

(b) Monthly Cluster Radar
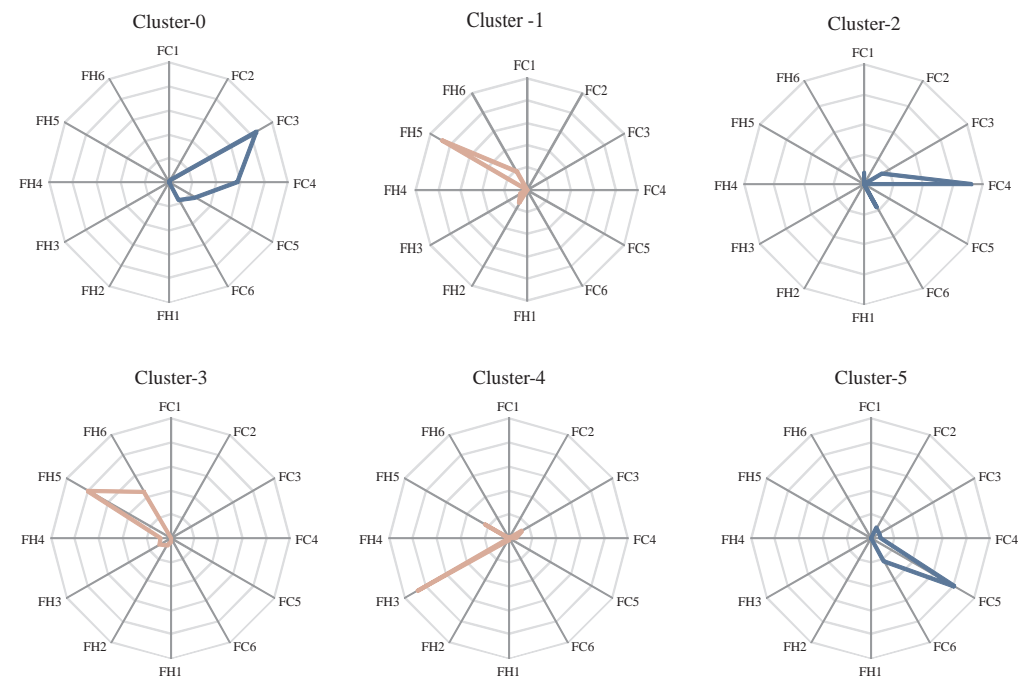

(c) Random Cluster Radar

Figure 11: Cluster wise feature distribution: (a) weekly TU data, (b) monthly TU data, and (c) randomly selected TU data 
(a)
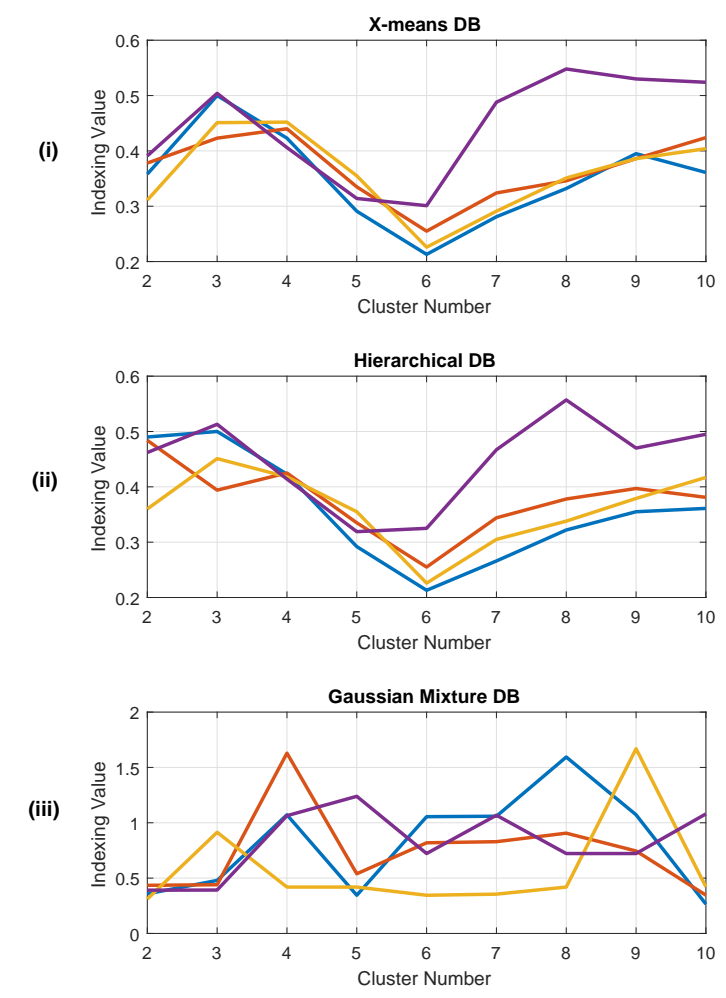

(b)
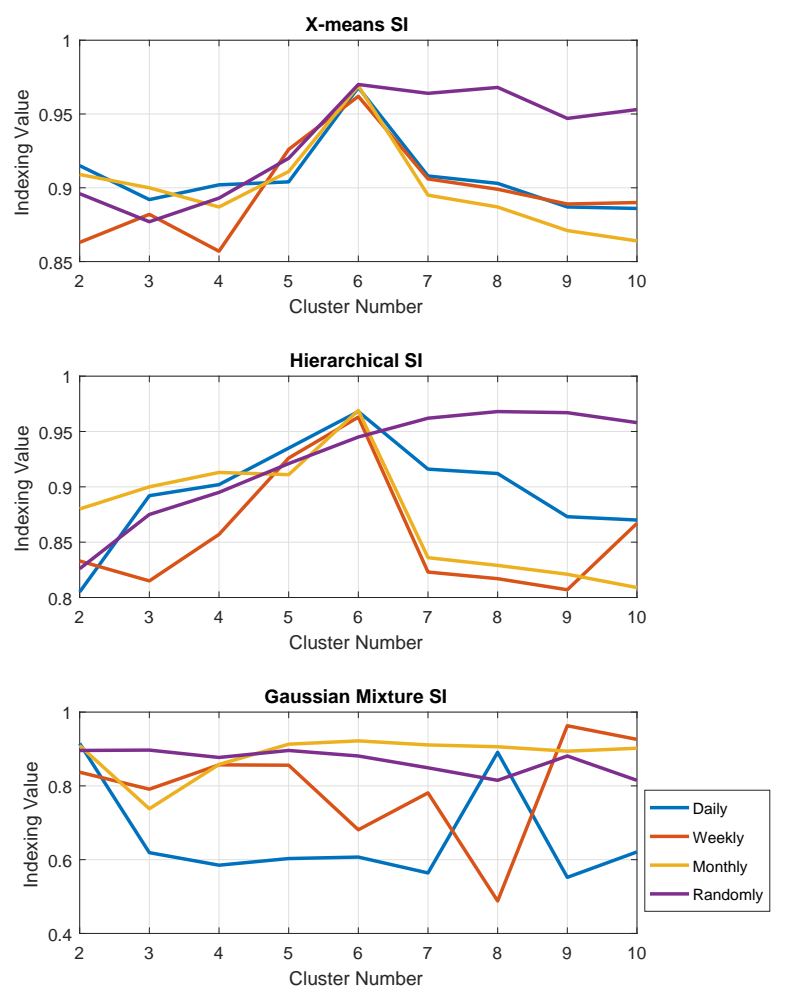

Figure 12: Clustering Validation and comparison

\subsection{Classification Result Analysis}

The clustering outcomes are used as pre-processing step for classification of TU data. Therefore, the TUs are labelled using the clustering outcomes to make an automated fault diagnosis and predictive system for buildings. In this phase the classification is performed by employing MCSVM, where the dataset is categorized and labelled into six different classes for diagnosing diverse behaviour of faulty and non-faulty TUs (tabulated in Table 2).

Here, the experiment is conducted by dividing the TU dataset in 10\%, 20\%, 30\% for training, and $90 \%, 80 \%, 70 \%$ respectively for testing purposes. The number of objects (NOB) or TUs are 656 . Statistical evaluation of performance parameters like, accuracy, precision, and recall are evaluated and recorded in Table 3. The highest precision and recall value obtained from $10 \%$ training and $90 \%$ testing data (marked in bold) in all the cases using MC-SVM. This algorithm achieved an excellent performance with $99.3 \%$ of precision in randomly selected data, which signifies a high positive predictive value for all predicted TUs. Also observed that the performance increased when the training data volume is being increased. The highest recall $96.3 \%$ is achieved in case of randomly selected data, which directs the relevant prediction of TUs with a very high accuracy. Thus, experimental result signifies that the algorithm can make fault predictions about TU behaviour with the help of very few amount (only 10\% is enough) of training data.

Confusion matrixes are set to concentrate on the type of TUs that are assigned in wrong category by the algorithm. Four confusion matrices are shown in Figure 13. The matrices are prepared based on the highest accuracy obtained by MC-SVM (where only 10\% training data are used). Each element in this matrix is the number of test items with true class in row wise and predicted class in column wise. The correctly classified objects are plotted diagonally (marked in blue color) 
Table 3: Classification results by MC-SVM

\begin{tabular}{|l|l|lll|}
\hline \multicolumn{2}{|l|}{ Observations } & $10 \%$ & $20 \%$ & $30 \%$ \\
\hline \multirow{3}{*}{ Daily } & NOB & 656 & 595 & 536 \\
& Precision & $\mathbf{0 . 9 8 3}$ & 0.982 & 0.979 \\
& Recall & $\mathbf{0 . 9 2 1}$ & 0.913 & 0.897 \\
\hline \multirow{3}{*}{ Weekly } & NOB & 3262 & 2970 & 2672 \\
& Precision & $\mathbf{0 . 9 7 9}$ & 0.978 & 0.977 \\
& Recall & $\mathbf{0 . 9 0 5}$ & 0.902 & 0.896 \\
\hline \multirow{3}{*}{ Monthly } & NOB & 12944 & 11705 & 10643 \\
& Precision & $\mathbf{0 . 9 8 8}$ & 0.988 & 0.987 \\
& Recall & $\mathbf{0 . 9 4 3}$ & 0.942 & 0.938 \\
\hline \multirow{3}{*}{ Randomly } & NOB & 29744 & 27002 & 24339 \\
& Precision & $\mathbf{0 . 9 9 3}$ & 0.992 & 0.992 \\
& Recall & $\mathbf{0 . 9 6 5}$ & 0.964 & 0.964 \\
\hline
\end{tabular}

and misclassified objects are marked in light blue. Though the overall performance of the proposed algorithm is good, still some characteristics of TUs confound the classifier which causes the misidentification. It is clearly seen that the objects of class-1 are misidentified mostly in case of daily (Figure 13(a)) and class-2 in case of weekly (Figure 13(b)) analysis. In Figure 13(a) and 13(b), 6 and $(11+7+3+17)=38$ objects are misidentified because, the TUs belong to these classes are distinct by the cooling and heating temperature but similar in nature with respect to power. In case of monthly and randomly (Figure 13(c) and 13(d)) data, misclassifications occurred mostly from class-0, whereas this class holds the good behaving and distinct TUs with respect to temperature and power consumption. A huge number of objects belong to this class and numerically the differences between the objects are very small. Therefore, the classifier is over fitted and overreacted to the slight fluctuations in the feature values.

Predicted
\begin{tabular}{|l|c|c|c|c|c|c|}
\hline n=656 & Class 0 & Class 1 & Class 2 & Class 3 & Class 4 & Class 5 \\
\hline Class 0 & 33 & 2 & 0 & 0 & 0 & 0 \\
\hline Class 1 & 0 & 431 & 0 & 1 & 0 & 0 \\
\hline Class 2 & 0 & 2 & 58 & 0 & 0 & 0 \\
\hline Class 3 & 0 & 1 & 0 & 51 & 0 & 0 \\
\hline Class 4 & 0 & 0 & 4 & 0 & 35 & 0 \\
\hline Class 5 & 0 & 1 & 0 & 0 & 0 & 37 \\
\hline
\end{tabular}

(a) Daily TUs

\begin{tabular}{|l|c|c|c|c|c|c|}
\multicolumn{7}{|c|}{ Predicted } \\
\hline n=12944 & Class 0 & Class 1 & Class 2 & Class 3 & Class 4 & Class 5 \\
\hline Class 0 & 7550 & 6 & 2 & 7 & 8 & 21 \\
\hline Class 1 & 9 & 1279 & 12 & 3 & 3 & 0 \\
\hline Class 2 & 26 & 2 & 811 & 6 & 0 & 0 \\
\hline Class 3 & 6 & 1 & 1 & 838 & 2 & 1 \\
\hline Class 4 & 4 & 1 & 1 & 2 & 1079 & 0 \\
\hline Class 5 & 6 & 4 & 8 & 7 & 3 & 1235 \\
\hline
\end{tabular}

\begin{tabular}{|c|c|c|c|c|c|c|}
\hline \multicolumn{7}{|c|}{ Predicted } \\
\hline $\mathrm{n}=3262$ & Class 0 & Class 1 & Class 2 & Class 3 & Class 4 & Class 5 \\
\hline Class 0 & 176 & 2 & 11 & 1 & 1 & 1 \\
\hline Class 1 & 0 & 221 & 7 & 0 & 0 & 1 \\
\hline Class 2 & 1 & 2 & 1929 & 0 & 2 & 0 \\
\hline Class 3 & 0 & 0 & $\mathbf{0}$ & 220 & $\mathbf{0}$ & 7 \\
\hline Class 4 & 2 & 1 & 3 & $\mathbf{0}$ & 348 & 0 \\
\hline Class 5 & 0 & 0 & 17 & 0 & 8 & 301 \\
\hline
\end{tabular}

(b) Weekly TUs

\begin{tabular}{|c|c|c|c|c|c|c|}
\multicolumn{7}{|c|}{ Predicted } \\
\hline $\mathrm{n}=29744$ & Class 0 & Class 1 & Class 2 & Class 3 & Class 4 & Class 5 \\
\hline Class 0 & 14364 & 0 & 13 & 0 & 5 & 4 \\
\hline Class 1 & 0 & 3252 & 2 & 11 & 4 & 2 \\
\hline Class 2 & 23 & 2 & 2139 & 5 & 1 & 0 \\
\hline Class 3 & 46 & 8 & 0 & 4311 & 13 & 0 \\
\hline Class 4 & 24 & 0 & 2 & 11 & 3188 & 0 \\
\hline Class 5 & 21 & 1 & 6 & 2 & 6 & 2278 \\
\hline
\end{tabular}

Figure 13: Confusion matrix by MC-SVM

(1)

b the class number to an object based on the nearest distance of its neighbours. Here, $\mathrm{k}$ is chosen as 1 and 3 and the results are shown in Table 4 . Table 4 shows the precision and recall outcomes for $1 \mathrm{NN}$ and $3 \mathrm{NN}$ with $10 \%, 20 \%$ and $30 \%$ training samples. Thus, it is observed from the result table that $30 \%$ training sample is required for $1 \mathrm{NN}$ and $3 \mathrm{NN}$ to produce maximum precision and recall 
values, except in case of $1 \mathrm{NN}$ for daily analysis $20 \%$ training data is needed for high performance. Thus, it is determined that MC-SVM provides high precision and recall with less number of training data than KNN classifier.

Table 4: Precision and recall comparison of $1 \mathrm{NN}$ and $3 \mathrm{NN}$

\begin{tabular}{|l|l|ll|ll|ll|}
\hline \multirow{2}{*}{ Observations } & \multicolumn{2}{|c|}{$10 \%$} & \multicolumn{2}{c|}{$20 \%$} & \multicolumn{2}{c|}{$30 \%$} \\
\cline { 3 - 8 } & & $1 \mathrm{NN}$ & $3 \mathrm{NN}$ & $1 \mathrm{NN}$ & $3 \mathrm{NN}$ & $1 \mathrm{NN}$ & $3 \mathrm{NN}$ \\
\hline \multirow{3}{*}{ Daily } & NOB & 654 & 656 & 588 & 593 & 525 & 533 \\
& Precision & 0.945 & 0.916 & 0.957 & 0.944 & 0.949 & 0.961 \\
& Recall & 0.774 & 0.686 & 0.818 & 0.772 & 0.786 & 0.829 \\
\hline \multirow{3}{*}{ Meekly } & NOB & 3265 & 3274 & 2966 & 2964 & 2680 & 2697 \\
& Precision & 0.957 & 0.950 & 0.969 & 0.967 & 0.972 & 0.973 \\
& Recall & 0.818 & 0.792 & 0.863 & 0.855 & 0.877 & 0.877 \\
\hline \multirow{3}{*}{ Randhly } & NOB & 12942 & 12968 & 11693 & 11721 & 10600 & 10621 \\
& Precision & 0.968 & 0.967 & 0.971 & 0.975 & 0.974 & 0.976 \\
& Recall & 0.861 & 0.855 & 0.871 & 0.886 & 0.885 & 0.890 \\
\hline & NOB & 29758 & 29766 & 26912 & 26896 & 24411 & 24383 \\
& Precision & 0.969 & 0.970 & 0.974 & 0.976 & 0.979 & 0.977 \\
& Recall & 0.865 & 0.865 & 0.882 & 0.891 & 0.904 & 0.898 \\
\hline
\end{tabular}

The precision and recall values are plotted in Figure 14 to compare the performance of MC-SVM, $1 \mathrm{NN}$, and $3 \mathrm{NN}$ for $10 \%, 20 \%$, and $30 \%$ training set. Precision and recall are shown in blue and yellow colour respectively. It is observed that MC-SVM achieved higher precision and recall than $1 \mathrm{NN}$ and $3 \mathrm{NN}$ for all time spans. Therefore, the performance of the MC-SVM shows the effective diagnosis of faulty and non-faulty HVAC TUs is possible with the help of supervised learning.

The fault diagnosis results by MC-SVM algorithm for all the experimented TUs described above coincide with the fact that indicates this method employed for any types of faulty and non-faulty HVAC TUs diagnosis is effective.

\subsection{Discussion}

Real TU behaviour is analysed remotely in this work, where temperature set point and corresponding power consumption are considered as the parameters for characteristics. These parameters enable a story to form around the HVAC and its direct environment, i.e. providing real data on the building under test and enabling managers to investigate faulty HVACs not all HVACs. Three distinct fault and three non-fault clusters have been remotely identified and classified. From the AFDD results, it can be concluded that faulty behaviour can be automatically and remotely detected and understood. Subsequently useful and exact information can be delivered to those managing the TUs in question. Using this vital new information an investigator can then identify if it is the environment (set-point issues) or the particular HVAC creating the fault?

Additionally predictive fault finding can be achieved using the classification results presented, thus managers can ensure timely interventions. In some cases where a fault is assumed, in actual fact the TUs in question once checked are not "broken" but for example have had their temperature set points set too high or low for their environmental setting, making it difficult for those TUs to achieve set point temperature leading to "faulty" behaviour displays. Additionally, too narrow dead band settings (difference between high and low set points) ranges cause many potential faulty behaviour. External influences such as weather conditions, sunlight, photocopiers, fridges, manual set point changes (based on personal preferences), open windows, etc. are not considered for both the set point and dead band levels estimation. Usually set point changes in situations above can be triggered by the building occupants depending upon their desired comfort levels and the effect 
(a)

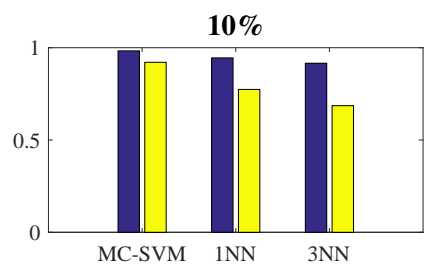

(b)

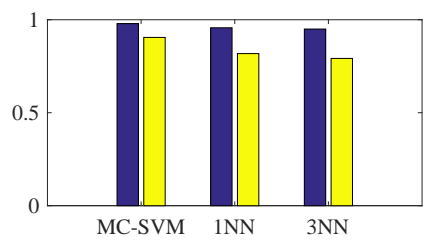

(c)

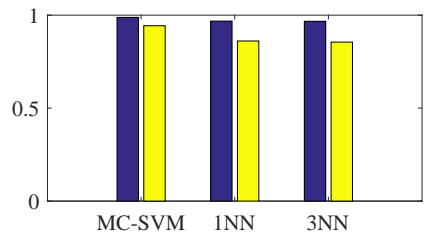

(d)

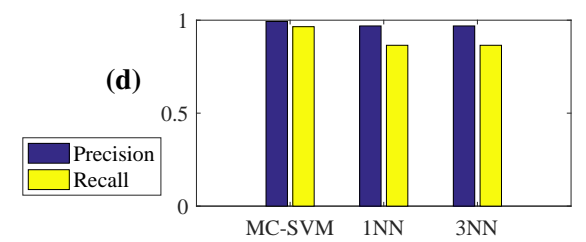

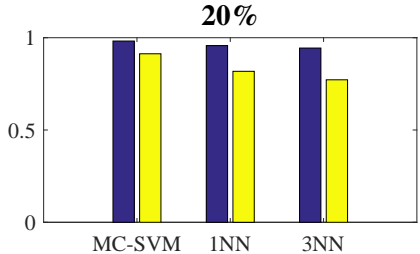
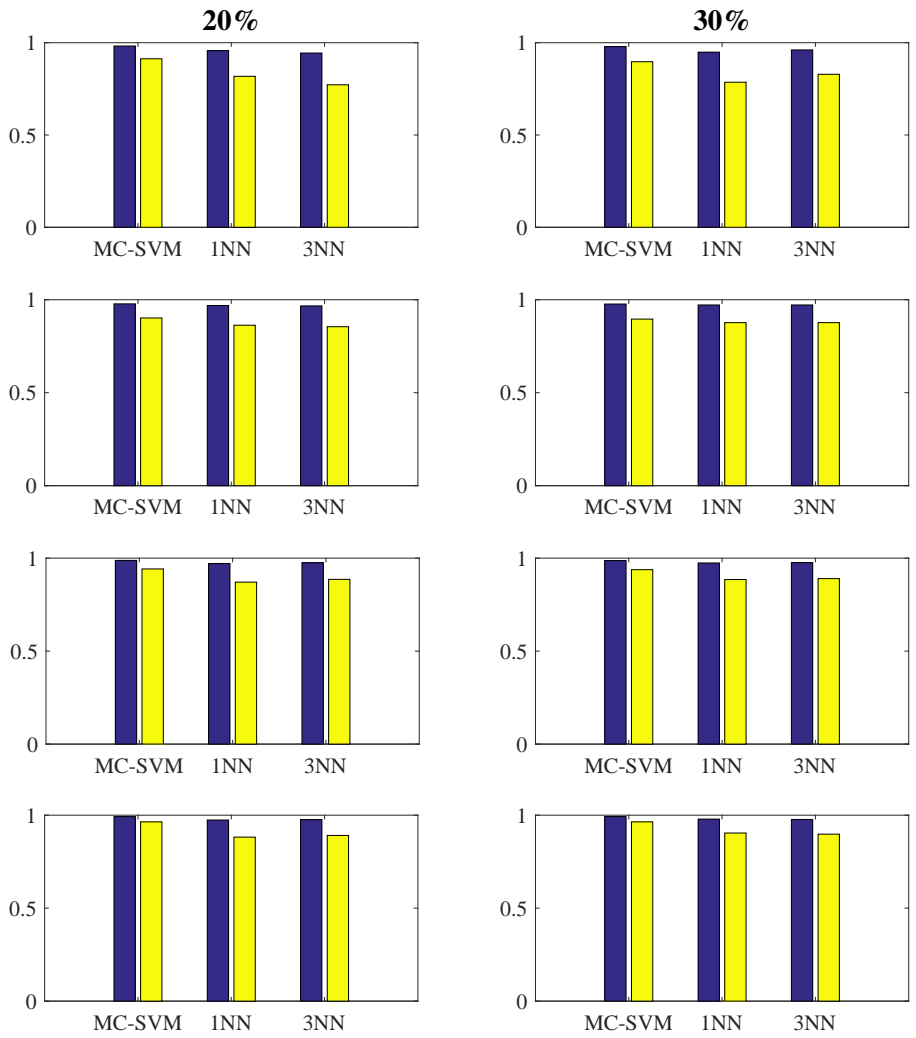

Figure 14: Precision and recall value analysis for TU data of (a) Daily, (b) Weekly, (c) Monthly, and (d) Randomly

of such request on TU behaviour is observable. Affected TUs subsequently demand excess power but will be unable to maintain the control strategies requested. Importantly the TUs in this case are not always defective but simply trying to achieve unattainable goals. Thus, energy and OPEX savings can be quickly made by addressing these external issues and the AFDD proposed provides this assistance.

\section{Conclusion and Future Work}

A novel feature extraction technique is presented to extract temperature and power associated features from high-dimensional and unstructured TU data. These features provide a good approximation for the TU characteristics and are tested on large number of TUs. An unsupervised learning technique was employed to identify distinct TU behavioural patterns and discriminate the faulty and non-faulty TUs. Subsequently, a classification method was applied to predict and diagnose the faults from a building automatically. Due to the more realistic implementation i.e. lack of knowledge of sensor location, weather data, and the occupancy information, it is difficult to detect and diagnose the cause of faults effectively here, though the excellent accuracy of the proposed method signifies the real world effectiveness of the work.

This experiment has been tested on more than 39,000 TUs and gathered the ground truth information. Augmenting this work includes further tests on TUs where faults such as, improper dead bands and set point temperatures have been identified using AFDD and rectified. It could be used to predict faults on more recent data by using semi-supervised learning techniques. Further 
analysis will be based on the external and internal causes of the faults by the help of building experts towards fully remote fault diagnosis, so that, the quality research can provide useful and meaningful conclusions. Additionally, deep learning based methods will be explored for on-line training and testing purposes to enact AFDD in a real-time manner. The method developed and deployed for this paper is limited to a specific type of TU (Fan Coil Unit) of a single building, but it will be extended to different types of TUs such as air handling unit (AHU), variable air volume (VAV) and chilled beam etc.

\section{Acknowledgement}

This work has been carried out as a part of the Innovate UK funded research project (EP/M506734/1) Energy Management \& Analysis Exploiting Existing BEMS Infrastructure \& Data and the authors are grateful to the Demand Logic team for providing us with the BEMS data, verification, invaluable information and feedback on building services and related performance issues.

\section{References}

[1] D. Kolokotsa, The role of smart grids in the building sector, Energy and Buildings 116 (2016) 703-708.

[2] C. Talon, R. Martin, Next generation building energy management system - new opportunities and experiences enabled by intelligent equipment, Technical report, Navigant Research (2Q 2015).

[3] P. Usoro, I. Schick, S. Negahdaripour, An innovation-based methodology for hvac system fault detection, Journal of dynamic systems, measurement, and control 107 (4) (1985) 284-289.

[4] D. Anderson, L. Graves, W. Reinert, J. Kreider, J. Dow, H. Wubbena, A quasi-real-time expert system for commercial building hvac diagnostics, ASHRAE Transactions (American Society of Heating, Refrigerating and Air-Conditioning Engineers);(USA) 95 (CONF-890609).

[5] S.-H. Cho, H.-C. Yang, M. Zaheer-Uddin, B.-C. Ahn, Transient pattern analysis for fault detection and diagnosis of hvac systems, Energy Conversion and Management 46 (18) (2005) 3103-3116.

[6] S. Shaw, L. Norford, D. Luo, S. Leeb, Detection and diagnosis of hvac faults via electrical load monitoring, HVAC\&R Research 8 (1) (2002) 13-40.

[7] T. Salsbury, A temperature controller for VAV air-handling units based on simplified physical models, HVAC\&R Research 4 (3) (1998) 265-279.

[8] T. Salsbury, R. Diamond, Fault detection in hvac systems using model-based feedforward control, Energy and Buildings 33 (4) (2001) 403-415.

[9] L. K. Norford, J. A. Wright, R. A. Buswell, D. Luo, C. J. Klaassen, A. Suby, Demonstration of fault detection and diagnosis methods for air-handling units, HVAC\&R Research 8 (1) (2002) $41-71$.

[10] P. Haves, M. Kim, Model-based automated functional testingmethodology and application to air-handling units (2005) 979-989. 
[11] S. Wu, J.-Q. Sun, A physics-based linear parametric model of room temperature in office buildings, Building and Environment 50 (2012) 1-9.

[12] S. Wu, J. Sun, Multi stage regression linear parametric models of room temperature in office buildings, Building and Environment 56 (2012) 69-77.

[13] L. H. Chiang, R. D. Braatz, E. L. Russell, Fault detection and diagnosis in industrial systems, Springer Science \& Business Media, 2001.

[14] J. Shiozaki, F. Miyasaka, A fault diagnosis tool for hvac systems using qualitative reasoning algorithms, in: Proceedings of the Building Simulation, Vol. 99, 1999.

[15] J. Schein, S. T. Bushby, N. S. Castro, J. M. House, A rule-based fault detection method for air handling units, Energy and buildings 38 (12) (2006) 1485-1492.

[16] J. Schein, S. T. Bushby, N. S. Castro, J. M. House, Results from field testing of air handling unit and variable air volume box fault detection tools.

[17] A. Dexter, J. Pakanen, et al., Demonstrating automated fault detection and diagnosis methods in real buildings, Technical Research Centre of Finland (VTT), 2001.

[18] J. Schein, S. T. Bushby, A hierarchical rule-based fault detection and diagnostic method for hvac systems, HVAC\&R Research 12 (1) (2006) 111-125.

[19] S. R. West, Y. Guo, X. R. Wang, J. Wall, Automated fault detection and diagnosis of hvac subsystems using statistical machine learning, in: 12th International Conference of the International Building Performance Simulation Association, 2011.

[20] E. Alpaydin, Introduction to machine learning, MIT press, 2014.

[21] C.-F. Chien, S.-L. Chen, Y.-S. Lin, Using bayesian network for fault location on distribution feeder, IEEE Transactions on Power Delivery 17 (3) (2002) 785-793.

[22] W.-Y. Lee, J. M. House, C. Park, G. E. Kelly, Fault diagnosis of an air-handling unit using artificial neural networks, Transactions American Society of Heating Refrigerating and Air Conditioning Engineers 102 (1996) 540-549.

[23] J. Liang, R. Du, Model-based fault detection and diagnosis of hvac systems using support vector machine method, International Journal of refrigeration 30 (6) (2007) 1104-1114.

[24] A. L. Dexter, D. Ngo, Fault diagnosis in air-conditioning systems: a multi-step fuzzy modelbased approach, HVAC\&R Research 7 (1) (2001) 83-102.

[25] D. Li, G. Hu, C. J. Spanos, A data-driven strategy for detection and diagnosis of building chiller faults using linear discriminant analysis, Energy and Buildings 128 (2016) 519-529.

[26] R. Isermann, Fault-diagnosis applications: model-based condition monitoring: actuators, drives, machinery, plants, sensors, and fault-tolerant systems, Springer Science \& Business Media, 2011.

[27] Y. Zhao, S. Wang, F. Xiao, Pattern recognition-based chillers fault detection method using support vector data description (SVDD), Applied Energy 112 (2013) 1041-1048. 
[28] A. Beghi, R. Brignoli, L. Cecchinato, G. Menegazzo, M. Rampazzo, F. Simmini, Data-driven fault detection and diagnosis for hvac water chillers, Control Engineering Practice 53 (2016) 79-91.

[29] A. Widodo, B.-S. Yang, Support vector machine in machine condition monitoring and fault diagnosis, Mechanical systems and signal processing 21 (6) (2007) 2560-2574.

[30] M. Dey, M. Gupta, M. Turkey, S. Dudley, Unsupervised learning techniques for HVAC terminal unit behaviour analysis, in: The 2017 IEEE International Conference on Smart City Innovations (IEEE SCI 2017), San Francisco, USA, 2017.

[31] K. J. Åström, T. Hägglund, Advanced PID control, ISA-The Instrumentation, Systems and Automation Society, 2006.

[32] Z. Huang, Extensions to the k-means algorithm for clustering large data sets with categorical values, Data mining and knowledge discovery 2 (3) (1998) 283-304.

[33] D. L. Davies, D. W. Bouldin, A cluster separation measure, IEEE transactions on pattern analysis and machine intelligence (2) (1979) 224-227.

[34] P. J. Rousseeuw, Silhouettes: a graphical aid to the interpretation and validation of cluster analysis, Journal of computational and applied mathematics 20 (1987) 53-65.

[35] K. Crammer, Y. Singer, On the algorithmic implementation of multiclass kernel-based vector machines, Journal of machine learning research 2 (Dec) (2001) 265-292.

[36] S. P. Rana, M. Dey, H. Siddiqui, G. Tiberi, M. Ghavami, S. Dudley, UWB localization employing supervised learning method, in: IEEE International Conference on Ubiquitous Wireless Broadband ICUWB2017, Salamanca, Spain, 2017.

[37] G. Bello-Orgaz, J. J. Jung, D. Camacho, Social big data: Recent achievements and new challenges, Information Fusion 28 (2016) 45-59.

[38] M. Zaharia, M. Chowdhury, M. J. Franklin, S. Shenker, I. Stoica, Spark: Cluster computing with working sets., HotCloud 10 (10-10) (2010) 95.

[39] R. S. Xin, J. Rosen, M. Zaharia, M. J. Franklin, S. Shenker, I. Stoica, Shark: Sql and rich analytics at scale, in: Proceedings of the 2013 ACM SIGMOD International Conference on Management of data, ACM, 2013, pp. 13-24.

[40] Á. B. Hernández, M. S. Perez, S. Gupta, V. Muntés-Mulero, Using machine learning to optimize parallelism in big data applications, Future Generation Computer Systems, 2017, Elsevier.

[41] I. H. Witten, E. Frank, M. A. Hall, C. J. Pal, Data Mining: Practical machine learning tools and techniques, Morgan Kaufmann, 2016.

[42] Z. Zhou, C. Wen, C. Yang, Fault isolation based on k-nearest neighbor rule for industrial processes, IEEE Transactions on Industrial Electronics 63 (4) (2016) 2578-2586. 